\title{
¿Que nadie te quite la palabra! Estrategias para aumentar la disposición a comunicarse en el aula de ELE
}

DAYANE MÓNICA CORDEIRO

Universidade Federal de Pernambuco

dayane.cordeiro@ufpe.br

\begin{abstract}
Resumen: En el aula de lenguas extranjeras, los alumnos disponen de un repertorio lingüístico en proceso de desarrollo, que les impide muchas veces, expresar todo su potencial comunicativo. $\mathrm{Y}$ es justo en este contexto, que se observa el impacto más significativo de las variables personales. La decisión de comunicarse que asume el hablante siempre que tiene la oportunidad de hacerlo influye directamente en los logros en su competencia comunicativa en la lengua extranjera. En este sentido, conocer los componentes de la disposición a comunicarse, cómo generarla y mantenerla en el aula pueden ser la clave hacia una enseñanza/aprendizaje de la expresión y la interacción oral más efectiva. El objetivo de este artículo es ofrecer pautas didácticas y metodológicas para aumentar la disposición a comunicarse de los estudiantes a favor de un aprendizaje más efectivo, duradero y experiencial.
\end{abstract}

Palabras clave: Disposición a comunicarse, didáctica, enseñanza de español lengua extranjera, competencia comunicativa

Don't let anyone take your word for it! Strategies to increase the willingness to communicate in the ELE classroom

Abstract: In the foreign language classroom, students' linguistic repertoire is still being developed, which often prevents them from expressing their full communicative potential. Precisely in this context previous research shows that personal variables have the most significant impact. The decision to communicate that speakers assume whenever they have the opportunity to do so influences directly the achievements in their communicative competence in foreign languages. For this reason, knowing the components of the willingness to communicate, and how to generate and maintain it in the classroom can be the key to more effective teaching / learning of oral expression and interaction. The aim of this paper is to offer didactic and methodological guidelines to increase students' willingness to communicate in favor of more effective, lasting, and experiential learning.

Key words: Willingness to communicate, didactics, teaching Spanish as a foreign language, communicative competence

\section{Introducción}

Tanto los estudios teóricos como aquellos que se aplican a la enseñanza/aprendizaje de lenguas extranjeras (LE) señalan la competencia comunicativa como el objetivo último de este proceso Esta competencia implica la capacidad de utilizar la lengua con éxito mediante el reconocimiento de las características de la comunidad de habla y el respeto hacia las reglas lingüísticas de uso de la lengua (Canale, 1983). Asimismo, el aprendizaje 
de LE se concibe como un proceso sumamente interactivo, al considerar el habla como un acto comunicativo donde los participantes y la situación comunicativa ocupan un lugar destacado.

Entre las destrezas que componen la competencia comunicativa, este artículo se centra en el desarrollo de la expresión y de la interacción oral dada su complejidad didáctica. "La habilidad para comunicarse oralmente es el objetivo que más esfuerzo supone" (Baralo, 2000, p. 164, Kremers, 2000, p. 461). Esta afirmación, se fundamenta en las características de la interacción oral, donde se combinan las competencias lingüísticas, sociolingüísticas, pragmáticas e interculturales del usuario y también es la destreza que ofrece mayor rentabilidad en todas las facetas de la vida personal, académica o laboral (Núñez Delgado y Hernández Medina, 2011).

Las actuaciones orales imponen al hablante la superación de varios obstáculos a nivel lingüístico, personal y social. En primer lugar, el habla es parte de la identidad del hablante, y la fuerza de los actos comunicativos, son capaces de desvelar la identidad del emisor, influyendo de forma significativa en su autoconcepto como hablante competente y también en la valoración que otras personas harán de sus actos comunicativos (Arnold, 2011). Asimismo, el desarrollo de la competencia oral se considere como uno de los escenarios que produce mayores niveles de ansiedad, puesto que el alumno necesita ser capaz de sostener la comunicación por medio de un instrumento con el que no está totalmente familiarizado. El impacto de las variables afectivas en el proceso de adquisición de LE y en especial, en el desarrollo de las destrezas orales, es uno de los temas que está ganando protagonismo en los últimos años, "this means that SLA researchers do not have the option of ignoring the new psychological approaches, particularly because those represent some of the most promising future research directions". (Dörnyei, 2019:33).

Así pues, el estilo cognitivo, las actitudes hacia la lengua, la disposición a comunicarse, la influencia de los factores afectivos y los factores contextuales, son parámetros que han de tomarse en cuenta en el proceso de enseñanza/aprendizaje de una lengua extranjera desde la teoría a la práctica. Visto que la comunicación en el aula de lenguas extranjeras no es solo la meta, sino uno de los medios para lograr la competencia comunicativa (Arnold y Fonseca 2004:50).

Frente a este paradigma, y preocupados con una enseñanza que contempla la complejidad del tratamiento de las destrezas orales, este artículo se centra en el impacto de la disposición a comunicarse y de qué manera un aumento de esta disposición puede facilitar el aprendizaje. Por ello, se presentarán los elementos que componen esta teoría, cómo generar y mantener la disposición a comunicarse en el aula y finalmente, algunas claves didácticas capaces de ayudar a los docentes en la labor de favorecer que los alumnos alcancen la competencia comunicativa, desde la perspectiva cognitiva y afectiva.

\subsection{La disposición a comunicarse en una LE}

Los enfoques actuales de enseñanza de LE enfatizan que la exposición a la lengua de calidad, así como un mayor número de oportunidades de práctica, tanto dentro como fuera del aula, son las claves para que el alumno alcance la competencia comunicativa. Pero ¿qué pasa cuando el alumno dispone de situaciones comunicativas adecuadas en la lengua meta, pero prefiere no arriesgarse? Varios estudios señalan que los factores 
ambientales, contextuales, cognitivos, sociales y afectivos (Csizér y Dörnyei 2005; Dörnyei 1994, 2005, 2008; Horwitz 1986, 2001; MacIntyre y Gardner 1994; Arnold y Brown 2000, Arnold, 2000) afectan en la disposición del alumnado a participar de las situaciones comunicativas, sean ellas auténticas o generadas.

Estos factores se han combinado en la teoría de la"Disposición a Comunicarse" (DC) (Willingness to communicate, MacIntyre, 1999,2003, 2011; MacIntyre, Baker, Clemént, Conrod, 2001; MacIntyre, Dörnyei, Clemént, Noels, 1998; Yashima 2002). Los primeros estudios sobre la DC la definen como "una disposición para entrar en el discurso en un momento determinado con una persona o personas específicas utilizando la LE o la L2" (MacIntyre, 1998:547).

El concepto de disposición a comunicarse rápidamente se exportó al estudio de la adquisición de LE (al principio su objeto de estudio era la adquisición de segundas lenguas (L2) en contexto de inmersión). Con relación al ámbito de adquisición de LE, estos autores sostienen que el objetivo fundamental del proceso de enseñanza/aprendizaje de una LE debería ser la creación de la disposición a comunicarse en el alumno, puesto que el énfasis en la competencia comunicativa puede producir estudiantes técnicamente capaces de comunicarse, particularmente dentro del aula, pero puede ser que estos alumnos no estén dispuestos a hacerlo fuera de este entorno (MacIntyre et al., 1998). Es decir, es necesario vincular la importancia de fomentar condiciones que promuevan y desarrollen la disposición a comunicarse en la lengua meta si queremos que los alumnos alcancen la competencia oral.

Asimismo, muchos estudiantes, aunque son competentes en la LE pueden evitar la comunicación en la lengua meta y en este caso pierden la oportunidad de desarrollar el idioma a través de la comunicación auténtica (Dörnyei, 2005). Por consiguiente, es imprescindible animar a los alumnos con dificultades, y también a los que tienen altas capacidades a buscar y aprovechar las oportunidades de comunicación en la lengua meta, y eso depende de su disposición a comunicarse.

Por todo ello, el número de investigaciones sobre la disposición a comunicarse y su impacto en el proceso de aprendizaje de LE aumentó considerablemente y ha proporcionado varios indicadores de los factores que favorecen u obstaculizan el desarrollo de esta competencia. En líneas generales partimos de dos premisas para justificar las estrategias que aumentan la DC: por un lado, se estipula que una alta disposición a comunicarse se traduce en mayores oportunidades de práctica y uso auténtico de la lengua, lo que proporciona, a su vez, mejores resultados en el desarrollo de la competencia comunicativa (MacIntyre et al, 2017). Por otro lado, una alta disposición a comunicarse ayuda a que los alumnos tengan más autonomía en el proceso de aprendizaje, haciendo elecciones independientes para aprender y practicar el idioma a través de la comunicación sin la ayuda del profesor fuera del aula. De esta manera se aumentan inevitablemente sus oportunidades de aprendizaje (Kang, 2005:278).

Conocer estos factores es de suma importancia porque nos permite planificar el desarrollo de la expresión y de la interacción oral y actuar frente a las dificultades que serán encontradas en este recorrido. Por ello, se dedica un apartado a los elementos capaces de generar y mantener la DC. 


\subsection{Cómo generar y mantener la disposición a comunicarse en el aula de ELE}

La decisión de comunicarse se basa en una red de procesos a nivel cultural e individual que hacen que el alumno evite o acepte la comunicación a través de una interacción de influencias dependientes del contexto, es decir, la disposición a comunicarse es un fenómeno dinámico que cambia su intensidad dentro de cada evento comunicativo (MacIntyre et al., 2012). Por ello se puede afirmar que la decisión de interactuar en el aula de ELE o fuera de ella, está determinada por factores internos y externos que no son estables y sufren la influencia de varios elementos: los factores sociales, individuales y situacionales.

Empezamos por los factores sociales. Los actos de habla influyen de forma significativa en el autoconcepto como hablante competente del alumno y también en la valoración que los demás harán de sus actos comunicativos. Y nadie quiere sentirse amenazado si tiene la posibilidad de evitarlo. Cuanto menos equitativa es la relación social que nos une al interlocutor, menor será nuestra disposición a comunicarse con esta persona y cuanto mayor cercanía, menos expuesta estará nuestra imagen personal y normalmente hay un aumento de nuestra disposición a comunicarse, que obviamente está condicionada también por los factores individuales y situacionales.

Ahora centramos la atención a los factores individuales. El aprendizaje de una lengua extranjera se difiere de cualquier otro tipo de aprendizaje, porque pertenece al ámbito social y es parte de la identidad de los individuos (Williams y Burden, 1999:77). Esta combinación de factores hace que el aprendizaje de una lengua extranjera se considere el escenario de aprendizaje que genera mayores niveles de ansiedad (Rubio,2004). La ansiedad es el factor personal que obstaculiza en mayor medida el buen desarrollo de las destrezas orales. Por lo tanto, no es suficiente con ofrecer oportunidades para que los alumnos practiquen las destrezas comunicativas, es necesario crear un ambiente comunicativo en el aula capaz de aumentar el autoconcepto del alumno como hablante competente y a la vez, reducir sus niveles de ansiedad.

Finalmente, hay que considerar el impacto de los factores situacionales. Una vez que sabemos que los actos de habla en una lengua extranjera exponen la identidad del hablante, este puede rehusar hacerlo, hacerlo porque se le obliga, o hacerlo porque quiere (Arnold y De Vega, 2015:65). Esta disposición depende del contexto de habla, de más a menos formal, por ejemplo, si la intervención se dará en una conferencia, una reunión o una charla y también el tipo de interlocutor, si es un desconocido, un conocido, o un amigo. Es decir, a nivel situacional, la ambigüedad de los actos comunicativos reduce la disposición a comunicarse, por ello, aunque el alumno tiene interés en seguir la conversación, acaba limitándola o simplificándola.

Una vez que conocemos estos elementos y tenemos en cuenta que los tres actúan de manera interrelacionada y fluctúan durante todo el acto comunicativo, desde el momento que el emisor desarrolla la idea, hasta el momento que se da la retroalimentación entre emisor y receptor, cabría visualizar la comunicación como un continuum, cuanto más nos acercamos a una de las extremidades, menor será la disposición a comunicarse y cuanto más nos acercamos al otro extremo, mayor será este deseo.

El éxito en la comunicación viene condicionado por varios factores, para comprenderlos, nos centraremos en su forma más prototípica: la conversación y los elementos que influyen en este registro (Briz,2010:26) y relacionarlos con los elementos que influyen en 
la disposición a comunicarse. En este sentido, las intervenciones comunicativas dependen de:

1. Las relaciones vivenciales de proximidad

2. La cotidianidad temática de la conversación

3. Nuestras relaciones sociales con los interlocutores

4. El marco interaccional

5. Si disponemos o no de tiempo de planificación

6. Si el tono de la conversación es más o menos formal

7. Y finalmente, el fin interpersonal de la comunicación.

Cuantas más condiciones se cumplan, mayor disposición a comunicarse en la lengua meta. Por lo tanto, no es suficiente con ofrecer oportunidades para que los alumnos practiquen las destrezas comunicativas, es necesario crear un ambiente comunicativo en el aula y conocer los factores que favorecen o bloquean la comunicación a nivel lingüístico y personal.

Una vez que ya se presentaron los elementos que influyen en la disposición a comunicarse y las características de la conversación, nos centraremos en las alternativas didácticas utilizadas para aumentar la DC. Con este objetivo varios estudios se centraron en los elementos que pudieran guardar relación con la disposición a comunicarse y que pudieran fomentarse a nivel didáctico. Se han desarrollado diferentes modelos a partir de estos elementos y el modelo más conocido y con mejores resultados prácticos, es el modelo piramidal de MacIntyre, Clément, Dörnyei y Noels 1998.

Los autores organizaron los elementos que favorecen la DC en un modelo piramidal compuesto por 6 niveles que indica que estrategias hay que seguir para llegar al vértice de la pirámide, que sería la actuación en la lengua meta. Este es el modelo que sirvió como inspiración para elaborar las claves didácticas que se ofrecen en este artículo. La elección de este modelo frente a otros se dio porque ha sido probado empíricamente en diferentes contextos y con diferentes lenguas y los resultados de las investigaciones coinciden en que si se quiere aumentar y mantener la disposición a comunicarse es importante tener en cuenta variables lingüísticas, sociales, psicológicas y afectivas que se traducen en: (MacIntyre et all. 2014)

- Las relaciones interpersonales en el aula y fuera de ella

- Los factores de personalidad del alumno

- El impacto comunicativo a nivel afectivo

- La motivación intrínseca y extrínseca

- Actitudes hacia la lengua

- Autopercepción de la competencia comunicativa

Una vez detectadas y analizadas estas influencias, la mayor parte de ellas, son modificables. Por ello, los profesores pueden manipular las condiciones del aula y aprovechar la disposición a comunicarse de los estudiantes para optimizar la interacción entre ellos. Las estrategias didácticas más efectivas para favorecer la DC consisten en elegir temas de conversación familiares e interesantes (Cao, 2014; Peng, 2012), proporcionar corrección de errores después de la intervención, no durante su desarrollo (Zarrinabadi, 2014), dar retroalimentación adecuada a los estudiantes (Pishghadam y 
Khajavy, 2014) y usar un comportamiento amistoso y de apoyo (Kang, 2005; Zarrinabadi, 2014). En resumen, si somos capaces de aumentar la satisfacción experimentada durante el proceso de aprendizaje, haciendo que el alumno disfrute más, el aprendizaje y la DC se incrementan, y los niveles de ansiedad serán más bajos.

A continuación, se presenta una infografía que resume y explica los niveles del modelo piramidal y que puede servir como una guía para aquellos profesores que desean aumentar la disposición a comunicarse de sus alumnos, a favor de un desarrollo de la expresión y de la interacción oral más exitoso.

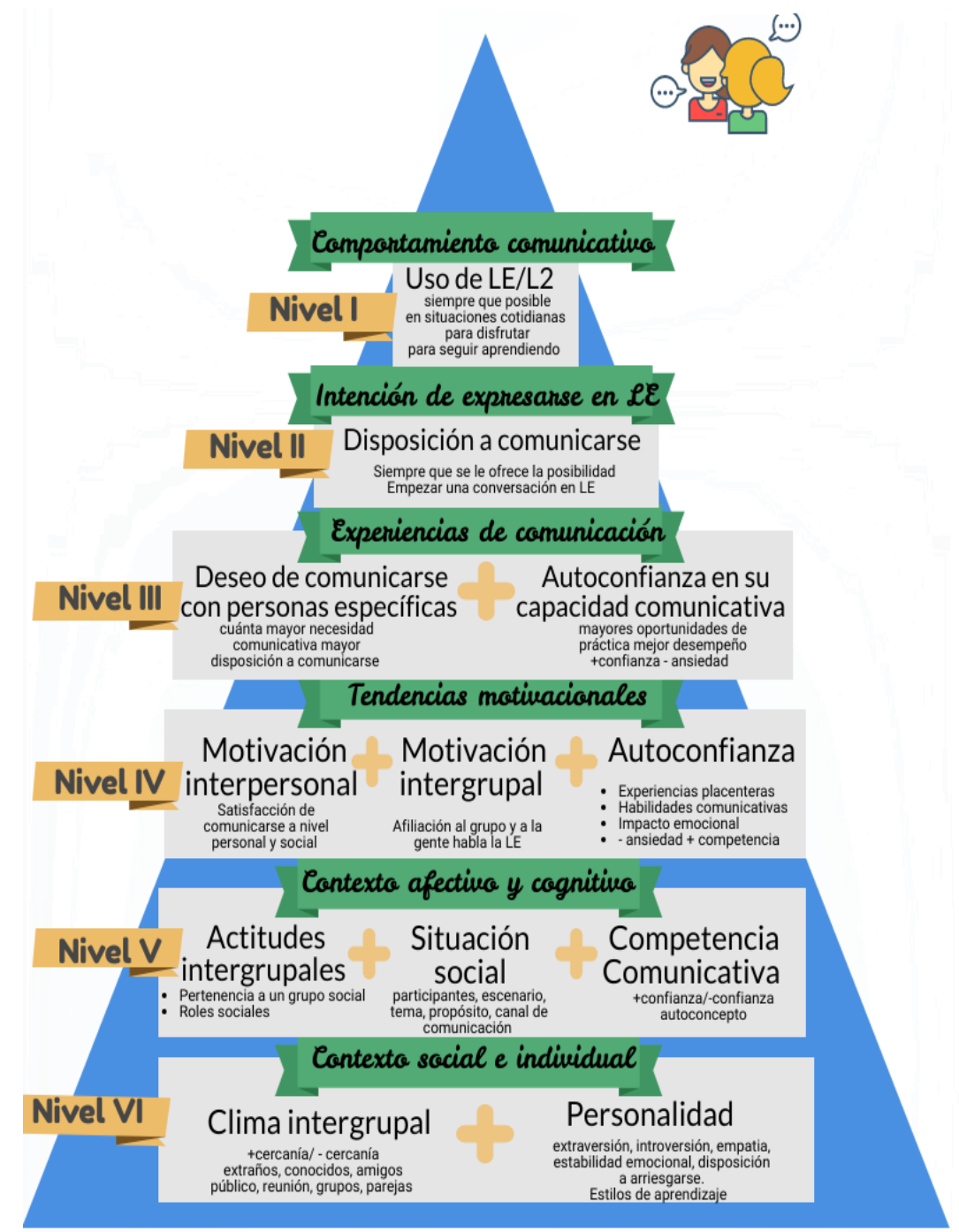

Imagen 1. Modelo piramidal de disposición a comunicarse a partir de (MacIntyre, Burns y Jesome, 2011:83) con la interpretación didáctica de Dayane Cordeiro

\section{Que nadie te quite la palabra: claves didácticas para aumentar la Disposición a comunicarse en el aula de ELE}

Presentados los elementos de la disposición a comunicarse y los factores que la favorecen desde la didáctica, en esta última parte, presentamos las claves prácticas desde el modelo piramidal. Este modelo se divide en seis niveles, y cuando el alumno es capaz de superar los cuatro primeros, alcanza los niveles más altos que sería la intención de expresarse en la lengua meta y el comportamiento comunicativo, es decir el uso efectivo de la lengua 
meta siempre que posible. En la práctica, ser capaz de desarrollar los niveles del modelo piramidal implica en una mayor autonomía comunicativa, mayor seguridad en practicar el idioma dentro y fuera del aula, y como resultado final, mejores resultados en el desarrollo de la competencia comunicativa. A continuación, se explican cada uno de estos niveles y se proponen algunas actividades efectivas para su desarrollo:

\section{Nivel VI del modelo piramidal: contexto social e individual}

La estrategia propuesta para superar el sexto nivel del modelo piramidal es cultivar la cohesión grupal. El grupo puede influir de manera positiva o negativa en las actitudes de los participantes hacia el proceso de aprendizaje de una LE y también aumentar o reducir las oportunidades de habla en el aula. Para ello, es necesario diseñar experiencias orientadas a crear y mantener un clima de aceptación y cooperación para un aprendizaje significativo. (Dörnyei, 2008). Facilitar la interacción alumno/alumno es el primer pilar para aumentar la disposición a comunicarse.

Las relaciones entre los alumnos con sus compañeros de clase, la manera en que ellos interactúan entre sí y la sensación de que los compañeros son un apoyo durante el proceso de aprendizaje, repercuten en una mayor o menor DC durante el desarrollo de las actividades de clase. Cuando llegamos a un nuevo grupo y principalmente cuando necesitamos interactuar utilizando un instrumento de comunicación que se está desarrollando, además de la barrera lingüística, los factores personales como la inseguridad, acostumbrarse a la personalidad de los demás, las relaciones interpersonales que surgirán y la aceptación de los compañeros, son elementos que hacen que el alumno decida participar de la comunicación o huir de ella.

En este sentido, trabajar en el aula diferentes dinámicas de grupo, pensar en proceso de formación de los grupos y trabajar con diferentes agrupaciones, puede aumentar la interacción entre los alumnos, una vez que, los patrones de interacción en clase se basen en el tamaño del grupo o número de participantes en la conversación y las relaciones de mayor a menor afinidad que los unen. A medida que aumenta el número de personas en una conversación, la seguridad de los participantes tiende a disminuir. En consecuencia, las actividades comunicativas funcionan mejor en parejas o grupos pequeños. El apoyo social y moral de los interlocutores también influye en la seguridad: cuando el oyente escucha atentamente, sonriendo o proporcionando respuestas activas, los interlocutores se sienten más seguros y tienen una mayor disposición a dar continuidad a la conversación.

A continuación, facilito algunas ideas prácticas que pueden ayudar a formar grupos efectivos capaces de favorecer la disposición a comunicarse:

- Formación de grupos heterogéneos con canciones: se eligen tres canciones diferentes, cuando suena la canción los alumnos se juntan con cuatro compañeros diferentes y deben memorizar los compañeros que coincidieron en cada canción. Se repite la misma dinámica con las demás, y al final el profesor elige una entre las tres canciones y los alumnos trabajan con los compañeros con los que coincidieron en esta.

- Repartir varios post-it en el aula. En cada post-it debes escribir una temática y los alumnos y alumnas deberán buscar el tema que les llama más la atención. Al final, se juntan los alumnos que manifestaron intereses en común a partir de la elección del post-it. 
- Con quien me uno: Cada alumno recibe el trocito de una canción, la cantidad de trocitos depende del número de participantes que deseamos para la actividad (3 partes de una misma canción, 4 o más), cada alumno empieza a cantar su canción y cuando coincide con otro compañero(a) con la misma, ellos se juntan y buscan a los demás compañeros.

\section{Nivel $V$ del modelo piramidal: contexto afectivo y cognitivo}

La estrategia propuesta para superar el quinto nivel del modelo piramidal es aumentar la confianza y las actitudes intergrupales. Uno de los primeros estudios sobre la influencia del contexto del aula en la DC realizado por Peng y Woodrow (2010) muestra que el entorno de aula afectivo aumenta la autoconfianza de los alumnos, influye en sus creencias y también en la motivación, y que la conjunción de todos estos elementos afectó a la DC de los investigados positivamente. Cuando el alumno se siente cómodo en el entorno del aula, sus características individuales pueden contribuir para que cada uno encuentre la manera de aprovechar sus oportunidades de práctica de la LE para aumentar su DC. Otro aspecto importante es partir de temas que fomentan el conocimiento de mundo compartido entre los estudiantes. Las tareas de aprendizaje influyen en la DC desde cuatro perspectivas: familiaridad, sensibilidad, interés en el tema y conocimientos previos (Cao, 2014). Las tareas interesantes y desafiantes provocan el compromiso de los alumnos en la clase, aumentando su DC (Pishghadam, et al.2014). Finalmente, a nivel lingüístico, es importante desarrollar las estrategias conversacionales compensatoria, como pedir aclaraciones, expresiones útiles para empezar una conversación, para superar carencias, pedir ayuda, alterar o ajustar el mensaje, etc. En este sentido, el entrenamiento de estrategias comunicativas para iniciar turnos haciendo preguntas y escuchando con atención para comentar lo que el interlocutor dice, así como saber reaccionar de forma adecuada, pueden ser muy útiles en las actividades interactivas, porque aumentan el nivel de control del alumno sobre la actividad.

A continuación, facilito algunas ideas prácticas que pueden ayudar a trabajar las estrategias conversacionales compensatorias a partir de un input afectivo y cognitivo:

- Que nadie te quite la palabra: en grupos de 3 o 4 personas elegir una persona que tendrá el objeto tabú que estará escondido en una bolsa oscura (objetos aleatorios del día a día), el alumno lo elige al azar, un compañero del grupo elige tres palabras tabúes que el portavoz no puede utilizar durante su descripción. A cada descripción, el grupo dice cuál es el objeto y quien manifiesta si las predicciones están correctas es el compañero que eligió la palabra tabú. Gana el grupo que advine el objeto tabú más rápido.

- Malentendido: un alumno se sienta de espaldas con el otro. Cada uno coge un objeto al azar y debe describir al compañero de que se trata sin decir su nombre. No se puede decir la palabra tabú y se pueden pedir aclaraciones. Es más divertido cuando los alumnos traen los objetos de casa, así además de adivinar el objeto, deben decir de qué compañero creen que podría ser. Otra alternativa es cambiar la palabra tabú, por poner los objetos a funcionar fuera de su contexto habitual (por ejemplo, una esponja puede servir para rascarse, como instrumento para realizar pintura, etc). 


\section{Nivel IV del modelo piramidal: tendencias motivacionales.}

La estrategia para este nivel será proporcionar actividades que aumenten la motivación a partir de la afiliación al grupo y a la gente que habla la lengua meta. Las investigaciones sugieren que varios factores influyen directa o indirectamente en la DC como, por ejemplo, la motivación, la autoconfianza (Yashima, 2002), la postura interaccional (Peng, 2015; Yashima, 2002) y las actitudes hacia la lengua y la cultura de la lengua meta (Khajavy et al., 2016; Yashima, 2002).

La motivación y la aptitud lingüística son dos de los elementos que utilizamos para explicar el éxito o el fracaso de un alumno en el proceso de adquisición de una LE, porque la motivación es «la combinación de esfuerzo más el deseo en alcanzar el objetivo de aprender la lengua, además de las actitudes hacia lo lingüístico» (Gardner, 1985:10). El impacto emocional llega al alumno mucho antes que el contenido y la motivación durante todo el proceso de aprendizaje se traduce en oportunidades de aprendizaje más significativas e influye en el esfuerzo que el estudiante estará dispuesto a invertir en la tarea. Por ello, si queremos superar el cuarto nivel del modelo piramidal, es importante proponer actividades con pegamento emocional, generar un clima afectivo de aula, que fomenta la autoestima y la confianza en uno mismo a partir de actividades en las que el alumno puede tener un buen rendimiento.

Los resultados más sólidos de estudios anteriores que relacionan la motivación con el aprendizaje indican que la autopercepción de la competencia comunicativa y una falta de ansiedad son los indicios más significativos de la DC (Elahi, Khajavy, MacIntyre, y Taherian, 2016) y ambos se alcanzan a partir de un ambiente de aula acogedor y la autoconfianza del alumno en su competencia comunicativa.

Un sentimiento de seguridad es capaz de aumentar la disposición a comunicarse en una LE porque atenúa la influencia negativa de los factores afectivos. Kang (2005) afirma que la seguridad parece estar determinada principalmente por los interlocutores. Su investigación demuestra que los alumnos se sienten inseguros cuando mantienen conversaciones con personas con las que nunca hablaron anteriormente y, por lo tanto, no conocen su nivel comunicativo en la lengua meta. Dado que la mayor parte de los intercambios comunicativos en el aula se realizan entre los alumnos, es importante ofrecerles oportunidades de interacción que aumenten su confianza hacia sus compañeros para que se sientan más seguros en establecer intercambios orales con ellos.

La dinámica de poder entre los hablantes también influye en la seguridad. Cuando el interlocutor se da cuenta de que los demás participantes de la comunicación ya habían establecido una relación mutua, se siente inhibido a continuar la conversación. Por ello, las dinámicas personales que hacen que los alumnos estrechen los lazos entre ellos pueden ser útiles para aumentar la seguridad a la hora de empezar una comunicación. Finalmente, cabe reflexionar sobre el efecto que las actividades producen en los estudiantes: se aprende lo que se siente, por ello, siempre que posible, es importante trabajar con actividades divertidas y que acerquen el alumnado a sus compañeros y a la cultura de la lengua meta.

A continuación, facilito una idea práctica que puede ayudar a trabajar la afiliación al grupo a partir de una actividad que aumenta la motivación del alumnado:

- Las identidades secretas: cada uno escribirá algo que le hace único, una curiosidad que los demás no sepan. Se comparte la identidad secreta con los 
compañeros de su grupo. A continuación, se las entregan a otro equipo y ellos entregaran sus identidades. Los grupos se ponen de acuerdo sobre quién es el dueño(a) de cada una de las identidades y anuncia al otro equipo sus predicciones. El equipo con el mayor número de respuestas correctas es el ganador.

\section{Nivel III del modelo piramidal: experiencias de comunicación}

Para el tercero y último nivel, utilizaremos como estrategia las actividades significativas con apoyo grupal. En los niveles anteriores, se hizo hincapié en la importancia de los temas elegidos, que las relaciones interpersonales contribuyen de forma significativa al compromiso de los estudiantes hacia el proceso de enseñanza/aprendizaje y que la relevancia de la motivación como elementos que influyen en una mayor DC.

En esta clave, nos centramos en los elementos interpersonales de la conversación. Cuando la situación comunicativa es de nuestro interés, estamos más dispuestos a correr riesgos y la interacción entre los alumnos suele potenciar la fluidez en lugar de la corrección, ayudando a que los alumnos estén más dispuestos a comunicarse en el aula. Durante las situaciones de habla auténticas o generadas, el alumno se enfrenta a una situación vulnerable que normalmente se realiza sobre la marcha, con altos niveles de inmediatez y poco tiempo de planificación, y la emoción sea ella positiva o negativa, es otro indicativo que causa que la disposición a comunicarse fluctúe durante todo el proceso.

Los estudiantes se muestran más entusiasmados en participar en las actividades cuando el tema les interesa y pueden aportar una experiencia personal que enriquece la conversación por ello, es importante involucrar al alumno en la elección de estos temas. En este sentido se pueden proponer lluvias de ideas, pedir que los alumnos propongan temas de conversación, etc. No obstante, hay que tener en cuenta que los temas de conversación que se reiteran en el aula ya no tienen el mismo impacto emocional. Otro aspecto que hay que tener en cuenta, es que en grupos numerosos hay muchos temas de interés distintos, en este sentido, se puede agrupar los alumnos por temas de interés para que todos dispongan de condiciones óptimas de práctica comunicativa que favorecen el aumento de la DC.

En resumen, elegir temas de conversación familiares e interesantes (Cao, 2014; Peng, 2012), proporcionar corrección de errores después de la intervención, no durante su desarrollo (Zarrinabadi, 2014), dar retroalimentación adecuada a los estudiantes (Pishghadam y Khajavy, 2014) y usar un comportamiento amistoso y de apoyo (Kang, 2005; Zarrinabadi, 2014). Asimismo, el alumno necesita reflexionar sobre cómo se llevan a cabo los actos comunicativos, conociendo los patrones de interacción esperados.

Finalmente, potenciar el uso de estrategias facilitadoras como desambiguar significados a partir del contexto, relacionar información de fuentes diversas, anticipar contenidos, ayuda a que el alumno alcance la confianza necesaria para participar en las situaciones comunicativas. Que disminuya el esfuerzo al utilizar la lengua meta, depende de la familiaridad del estudiante con las experiencias de comunicación compartidas, y cada experiencia de comunicación permite a los alumnos negociar y renegociar su sentido del yo con relación al mundo social.

A continuación, facilito una idea práctica que puede ayudar a trabajar una experiencia de comunicación capaz de llegar a la dimensión comunicativa y afectiva: 
- Regala experiencias a la carta: esta es una actividad inspirada en las "Cajas de experiencias" que se pueden regalar y que se venden en diferentes almacenes. Los alumnos en grupos deben pensar en una experiencia que creen que otras personas querrían experimentar en su ciudad, por ejemplo, una visita al mercado central, probar alguna comida, visitar una exposición, dar un paseo, todo lo que se les ocurra a los alumnos. La experiencia se guardará en un sobre y en el verso el grupo elegirá un título para esta experiencia. El profesor puede colgar las experiencias en la pared o ponerlas encima de una mesa y los demás grupos o alumnos eligen una. Otra variación, sería que un integrante del grupo o todo el grupo promocione su experiencia a los demás grupos. El objetivo final, es que los alumnos que idealizaron la experiencia la vivan con el grupo o con el alumno(a) que la eligió, favoreciendo una comunicación auténtica e interpersonal a partir de una experiencia compartida.

\section{Consideraciones finales}

En este artículo, nos hemos preocupado en describir de qué manera la disposición a comunicarse influye en el desarrollo de la expresión y de la interacción oral en el aula de LE y a partir de estar premisas, señalar las condiciones que pueden favorecer la DC. El enfoque comunicativo concede una mayor relevancia a todos los factores que influyen en la adquisición de una LE, desde los elementos lingüísticos hasta los elementos relacionados al ámbito intrapersonal e interpersonal. Para el profesor, trabajar las destrezas orales en el aula es una tarea compleja, dado que es justo en el desarrollo de estas destrezas donde se nota el impacto de los factores personales y afectivos con mayor intensidad.

Por ello, partir de un enfoque que contempla al alumno desde su complejidad es útil en el sentido de que nada es más personal y complejo que expresarse con un recurso lingüístico que aún está en proceso de desarrollo y desvela la identidad del hablante. Hasta este punto hemos visto como la disposición a comunicarse es un elemento clave en el desarrollo de la competencia oral y que en la práctica podemos combinar una serie de estrategias socioafectivas y contextuales para alcanzar mejores resultados, alabados por los estudios teóricos y por la práctica del aula.

En este sentido, el repaso de las estrategias que aumentan la DC muestra que tener un mayor control sobre el proceso de aprendizaje, sobre los factores afectivos que influyen en ello y, principalmente, sobre las tareas que diseñamos con objetivos comunicativos pueden reducir la ansiedad y conducir a un nivel más alto de disposición a comunicarse. Los alumnos sienten el impacto afectivo del profesor y este impacto repercute en su disposición a enfrentarse a los desafíos que supone la interacción, aumentando o disminuyendo su disposición a comunicarse. Es decir, si somos capaces de aumentar la satisfacción experimentada durante el proceso de aprendizaje, haciendo que el alumno disfrute más, el aprendizaje y la DC se incrementan, y los niveles de ansiedad son más bajos.

También se ha insistido en que las actividades de desarrollo de la expresión y de la interacción oral no deben centrarse solo en objetivos lingüísticos, sino que es importante ofrecer al alumno una experiencia gratificante capaz de implicarlo desde la dimensión intelectual, racional y afectiva. En esta línea, el profesor necesita crear redes de interacción en el aula en las que el vehículo sea la lengua meta, y el combustible, los 
factores afectivos. Para ello, las actividades han de presentarse a través de tareas posibilitadoras, que proporcionan al alumno material lingüístico suficiente para tener éxito en la tarea. De esta manera, disponer de estrategias de comunicación, estrategias que aumentan su disposición a comunicarse utilizando la lengua meta y una atmósfera de aula de confianza ayuda a disminuir el impacto negativo que las actividades orales pueden producir en algunos alumnos.

Finalmente, todos los profesores deberían ser conscientes de que las elecciones metodológicas y didácticas que tomen ejercerán una fuerte influencia en el aula. Las actividades y las prácticas docentes no son vehículos neutrales, y todos los elementos influyen en el rendimiento comunicativo del alumno. Si aspiramos a que nuestros alumnos disfruten de los beneficios de una metodología afectiva y efectiva capaz de producir el desarrollo de la expresión y de la interacción oral, es necesario conectar los componentes de la expresión y de la interacción oral y fue justo lo que se propuso en este artículo.

\section{Bibliografía}

ARNOLD, J. (2000a). «La Dimensión Afectiva en el Aprendizaje de Idiomas». Cambridge: Cambridge University Press.

ARNOLD, J. (2011). «Attention to Affect in Language Learning», Anglistik. International Journal of English Studies, 22/1, pp. 11-22.

ARnold, J. y Brown, D. (2000). «Mapa del terreno». En Arnold, J (ed), La dimensión afectiva en el aprendizaje de idiomas (pp. 19-41). Cambridge: Cambridge University Press.

ARnOld, J. y De VeGA, C. J. (2015). «Facilitando la expresión y la interacción oral en la clase de español como L2». Ávila, J. (coord.). Didáctica de la emoción. Revista MarcoELE, núm. 21, pp. 62-75.

ARNOLD y FONSECA (2004). «Reflexiones sobre aspectos del desarrollo de la competencia comunicativa oral en el aula de español como segunda lengua». En Lorenzo, F. (ed), El desarrollo de la competencia lingüística y comunicativa en el aprendizaje de español como L2 (pp. 45-60). Madrid Edinumen.

BARALO, M. (2000). «El desarrollo de la expresión oral en el aula de ELE». En Carabela (ed.), El desarrollo de la expresión oral en el aula de E/LE (pp. 5-36). Madrid: SGEL.

BRIZ, A. (2010). «El registro como centro de la variedad situacional. Esbozo de la propuesta del grupo Val.Es.Co sobre las variedades diafásicas». En Fonte, I.; Rodríguez Alfano, (compiladoras), Perspectivas dialógicas en estudios del lenguaje (pp. 21-56). León: Universidad Autónoma de Nuevo León, Nuevo México.

CANALE, M. (1983). «From communicative competence to communicative language pedagogy». En J. C. Richards y R.W. Schmidt (eds.), Language and Communication (pp. 2-27). Londres: Longman.

CAO, Y. (2011). «Investigating Situational Willingness to Communicate Within Second Language Classrooms from an Ecological Perspective», System 39, pp. 468-479.

CSIZÉR, K. y DÖRNYEI, Z. (2005). «Language learners' motivational profiles and their motivated learning behavior», Language Learning, 55 (4), pp. 613-659. 
DÖRNYEI, Z. (1994). «Understanding L2 motivation: On with the challenge!», Modern Language Journal, 78, pp. 515-523.

DÖRNYEI, Z. (2005). The psychology of the language learner: Individual differences in second language acquisition. Mahwah, NJ: Lawrence Erlbaum.

DÖRNYEI, Z. (2008). Estrategias de motivación en el aula de lenguas. Madrid: Editorial UOC.

DÖRNYEI, Z. (2019). «Psychology and language learning: The past, the present and the future», Journal for the Psychology of Language Learning, 1(1), pp. 27-41.

ElAhi, M., KhAJAVY, G. H., MACINTYRe, P. y TAHERIAN, T. (2016). «A meta-analysis of L2 willingness to communicate and its three high-evidence correlates». Manuscript submitted for publication.

HoRwITZ, E. K. (1986). «Preliminary evidence for the reliability and validity of a foreign language anxiety scale», TESOL, pp. 559-562.

Horwitz, E. K. (2001). «Language Anxiety and Achievement», Annual Review of Applied Linguistics, 21 (1), pp. 112-126.

KANG, S. J. (2005). «Dynamic emergence of situational willingness to communicate in a second language», System 33 (2), pp. 277-292.

KREMERS, M. F. (2000). «El uso de las estrategias de aprendizaje en la expresión oral», ¿Qué español enseñar? Norma y variación lingüisticas en la enseñanza del español extranjeros. Actas del XI Congreso Internacional de ASELE en Zaragoza [en línea]. Centro Virtual Cervantes, 2000, pp. 461-470. [Fecha de consulta:10/02/2020]

MACINTYRE, P. D. (1999). "Language anxiety: A review of the research for language teachers»». En Young, D. J. (ed.), Affect in foreign language and second language teaching: A practical guide to creating a low-anxiety classroom atmosphere (pp. 24-45). Boston, MA: McGraw-Hill.

MACINTYRE, P. D. (2007). «Willingness to communicate in the second language: understanding the decision to speak as a volitional process», The Modern Language Journal, 91 (4), pp. 564-576.

MACINTYRE, P. D. (2012). «The idiodynamic method: A closer look at the dynamics of communication traits», Communication Research Reports, 29, pp. 361-367.

Macintyre, P.D., Burns, C., \& JESSOME, A. (2011). «Ambivalence about communicating in a second language: A qualitative study of French immersion students' willingness to communicate», The Modern Language Journal, 95(1), pp. 81-96

Macintyre, P. D. y Mercer, S. (2014). «Introducing positive psychology to SLA», Studies in Second Language Learning and Teaching, 4, pp. 153-172.

MACINTYRe, P. D. y VInCZE, L. (2017). «Positive and negative emotions underlie motivation for L2 learning», Studies in Second Language Learning and Teaching, 7, pp. 61-88.

MACINTYRE, P. D. y VincZe, L. (2017). «Positive and negative emotions underlie motivation for L2 learning», Studies in Second Language Learning and Teaching, 7, pp. 61-88. 
MACINTYRE, P. D. y GARDNER, R. C. (1994). «The effects of induced anxiety on three stages of cognitive processing in computerized vocabulary learning», Studies in Second Language Acquisition, 1, 6, pp. 1-17.

Macintyre, P. D., Baker, S. C., Clément, R. y Conrad, S. (2001). «Willingness to communicate, social support and language learning orientations of immersion students», Studies in Second Language Acquisition, 23, pp. 369-388.

Macintyre, P., Clément, R., Dörnyei, Z. y Noels, K. (1998). «Conceptualizing willingness to communicate in a L2: A situated model of confidence and affiliation», Modern Language Journal, 82, pp. 545-562.

Macintyre, P. D. y GREGERSEN, T. (2012). «Emotions that facilitate language learning: The positive-broadening power of the imagination». Studies in Second Language Learning and Teaching, 2, pp.193-213.

NúÑEz Delgado, M. P. y Hernández MedinA, A. (2011). «La Interacción oral en la Enseñanza de Idiomas: aportaciones de una investigación sobre interrupciones conversacionales», Porta Linguarum: revista internacional de didáctica de las lenguas extranjeras, $\mathrm{n}^{\circ} .16,2011$, pp. 123-136.

Peng, J. y Woodrow, L. (2010). «Willingness to communicate in English: A model in the Chinese EFL classroom context», Language Learning, 60, pp. 834-876.

PishghadAM, R. y KHAJAvy, G. H. (2014). «Development and validation of the Student Stroke Scale and examining its relation with academic motivation», Studies in Educational Evaluation, 43, pp. 109-114.

Rubio AlcalÁ, F. D. (2004). La ansiedad en el aprendizaje de idiomas. Huelva: Publicaciones Universidad de Huelva.

ZARRINABADI, N. (2014). «Communicating in a second language: Investigating the effect of teacher on learner's willingness to communicate», System, 42, pp. 288-295.

Williams, M. y BuRden, R. (1999). Psicología para profesores de idiomas: Enfoque del constructivismo social. Cambridge University Press.

YASHIMA, T. (2002). «Willingness to Communicate in a Second Language: The Japanese EFL Context», The Modern Language Journal, 86 (1), pp. 54-66. 